\title{
BROADBAND IMPEDANCE CALCULATIONS OF THE TAN VACUUM CHAMBER FOR THE LARGE HADRON COLLIDER (LHC)*
}

\author{
D. Li ${ }^{\S}$, G. Lambertson, W. Turner, LBNL, 1 Cyclotron Road, Berkeley, CA, U.S.A.
}

\begin{abstract}
We report research studies on broadband impedance calculations of the TAN vacuum chamber for the Large Hadron Collider (LHC). The TAN is the zero degree neutral particle absorber and contains the transition from a single vacuum beam tube to two beam tubes. Single bunch collective instability induced by broadband impedance may limit the beam intensity of the LHC. The broadband impedance of each component in the machine needs to be studied carefully, and the total impedance be controlled below certain threshold. Due to the complexity of the TAN chamber geometry, the impedance calculation has to be performed using 3D MAFIA in the time domain. To verify numerical simulation results, a few examples with simple geometries have been studied, and compared with the results obtained by analytical formula and a wellknown 2D code ABCI. A 3D model for the TAN chamber is established to estimate the magnitude of longitudinal broadband impedance. Transverse broadband impedance has been studied numerically as well and will be discussed.
\end{abstract}

\section{INTRODUCTION}

Among the US efforts on the US-LHC Accelerator Project, Lawrence Berkeley National Laboratory (LBNL) is responsible for the design and construction of the neutral particle (TAN) and IR front quadrupole (TAS) absorbers [1]. Figure 1 is an exploded view of the TAN absorber.

\section{TAN exploded view}

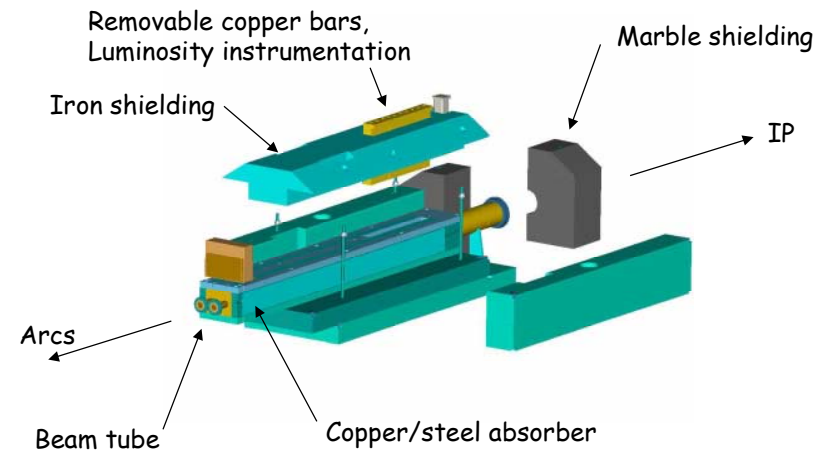

Figure 1: Exploded view of the TAN vacuum chamber

A horizontal mid plane section of the TAN design is shown in Figure 2 to illustrate more details of the

\footnotetext{
* Work supported by the U.S. Department of Energy under contract number DE-AC0376SF00098

§Email: Dli@1bl.gov
}

chamber. The TAN chamber contains transition from a single beam tube to two separate beam tubes and is symmetric from the middle plane. A single vacuum tube faces the IP and two beam tubes are away from the IP. The composite beam tube is manufactured from OFS copper. Dimensions of the TAN are shown in Figure 2 as well. A 3D view of the transition beam tubes is shown in Figure 3. The beam tube transition is the intersection of two cones with common base and separate apexes.

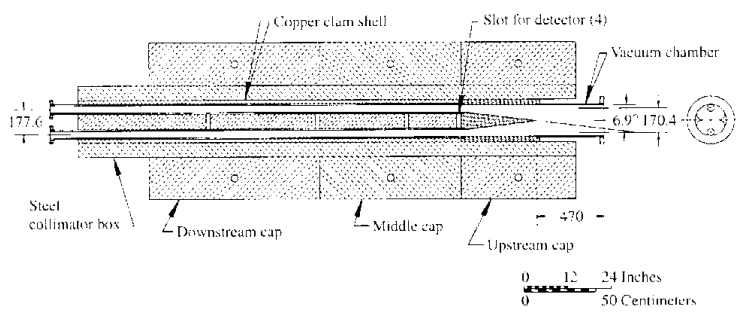

Figure 2: Horizontal mid plane section through the TAN Chamber.

In the design phase of the LHC, it is important to keep the broadband impedance seen by the beam to be below a certain impedance limit so that it will not be the limiting factor for having stable beams. Any discontinuity around the vacuum chamber (e.g. bellows, beam position monitors, RF cavities, tapers, vacuum valves, etc.) contributes to the global impedance of the machine, and needs to be evaluated carefully to make sure that it makes an acceptable small contribution to the total impedance budget. Impedance is a frequency domain description of the wakefield in the time domain. The wakefield is further categorized by its interaction time scale with the beam bunch as: short range wakefield (interaction with single beam bunch) and long range wakefield (interaction with the trailing beam bunches), which correspond to broadband and narrow band impedance in the frequency domain description, respectively. Therefore two aspects of the impedance need to be answered separately. For the broadband impedance, the short range wakefield needs to be calculated in order to find its low frequency (inductive) impedance, which is the main topic to be addressed in this paper. Narrow band impedance is associated with high Q resonance in the component, and the fields are often called "trapped modes". Trapped modes can be studied through either long range wakefield in the time domain or directly in the frequency domain simulation. In fact, we 
have performed the frequency domain simulations of the TAN chamber based on a 3D MAFIA model. The simulation results show that there is no trapped mode found in the TAN vacuum chamber for frequencies up to $2 \mathrm{GHz}$. In this paper, we concentrate on the discussions of the broadband impedance calculations based on the short range wakefield computation by the MAFIA in the time domain.

\section{TAN beam tube transition}

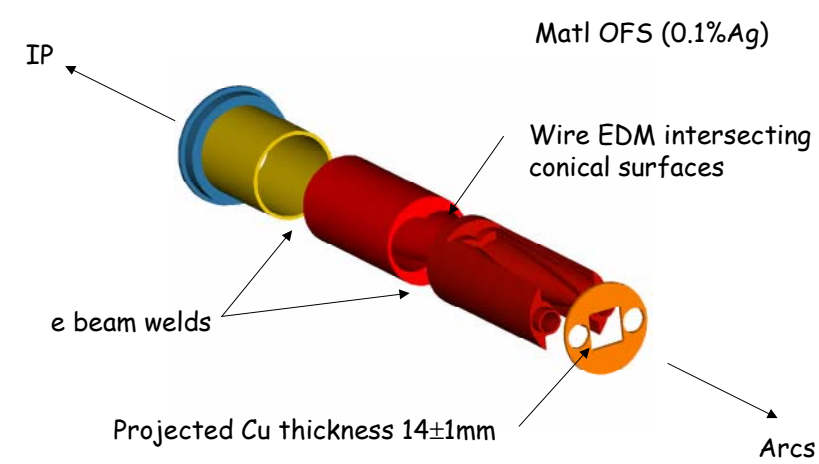

Figure 3: The TAN beam tube transition where two small beam tubes merge to a single large one at the IP.

Due to the geometric complexity of the TAN, 3D modeling is necessary. It turns out that the MAFIA code (T3) is the only existing code that is capable of simulating this $3 \mathrm{D}$ problem in the time domain. The wakefield is induced, computed and recorded by sending a Gauss distribution beam through the chamber. A fast Fourier transform (FFT) of the resulting wakefield normalized by the beam spectrum gives the impedance of the chamber. The low frequency response (inductive) of the chamber is obtained by measuring the slope of imaginary impedance at low frequency. To verify the MAFIA simulation results, we first bench mark the code by simulating a cylindrical beam pipe with a pillbox cavity in between, and comparing with the results obtained from an analytical formula [2] and another well-known 2D code, ABCI. Good agreements between the MAFIA and ABCI are achieved. A cylindrical taper, a rectangular wedge and the TAN chamber are simulated afterwards

\section{A CYLINDRICAL TAPER}

A cylindrical taper has been simulated to study how the low frequency response varies with the taper angle, as shown in Figure 3.

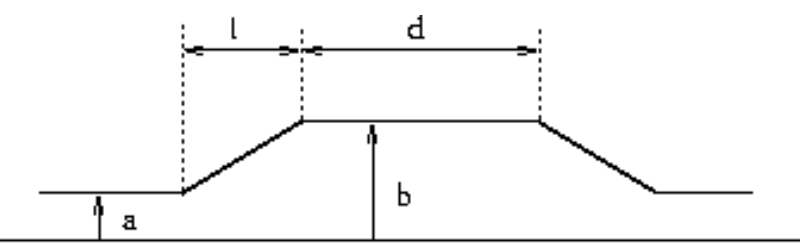

Figure 3: A cylindrical taper with $\mathrm{a}=2.6 \mathrm{~cm}, \mathrm{~b}=8.9 \mathrm{~cm}$,

\section{$\mathrm{d}=50 \mathrm{~cm}$ and 1 varies. If $\mathrm{l}=0$, the geometry becomes a} beam pipe with a cylindrical pillbox cavity

The 2D ABCI code was used to study the inductance variations with the taper angle. The inductance is plotted versus $l$, the length of the taper in Figure 4. As $l$ approaches to zero, the inductance increases and the taper eventually becomes a beam pipe with a simple cylindrical pillbox cavity where its inductance can be readily calculated by analytical formula,

$$
L=\frac{\mu_{0}}{2 \pi}(b-a) \ln \left(\frac{b}{a}\right)
$$

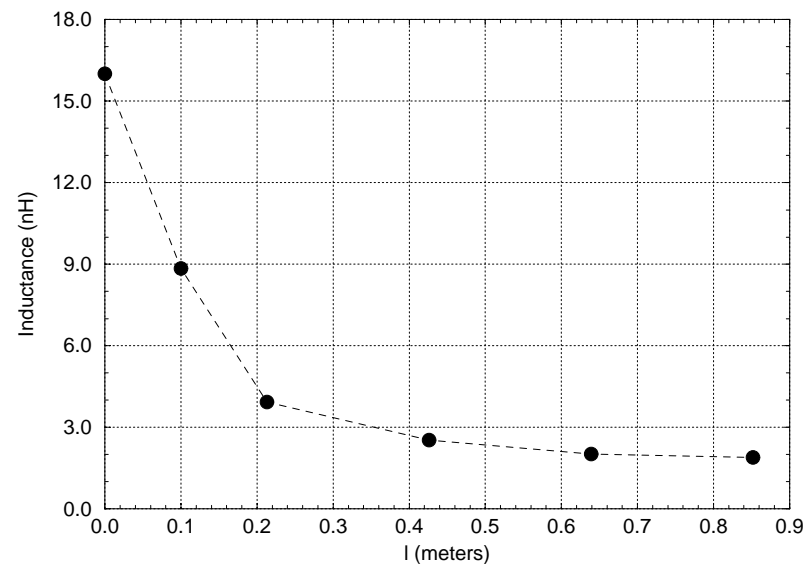

Figure 4: Inductance vs. $l$ (corresponding to taper angle changes), the results displayed by dots are simulated using the ABCI (2D) code.

The analytical formula yields $L=16 \mathrm{nH}$ which agrees very well with the $\mathrm{ABCI}$ simulation at $l=0$.

\section{THE LHC TAN VACUUM CHAMBER}

A 3D MAFIA model is established for the simulation, as shown in Figure 5 where only one half of the model (solid) is displayed. This model has been used for both the frequency and time domain simulations.

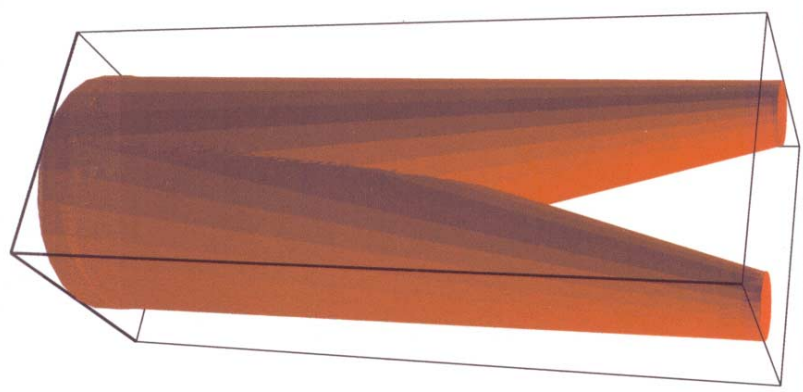

Figure 5: A 3D MAFIA model established for the time domain simulation. Only one half of the model is displayed here. The model has the same transition dimensions as the design for the TAN chamber shown in Figure 1. 
The longitudinal wakefield is computed by sending a Gauss distribution beam $\left(\sigma_{\mathrm{s}}=2.5 \mathrm{~cm}\right)$ through the center of one of the small beam pipes. The resulting longitudinal wakefield is recorded for about 3 meters. A FFT of the wakefield normalized by the beam spectrum yields the impedance, and the results are plotted against frequency as shown in Figure 6.

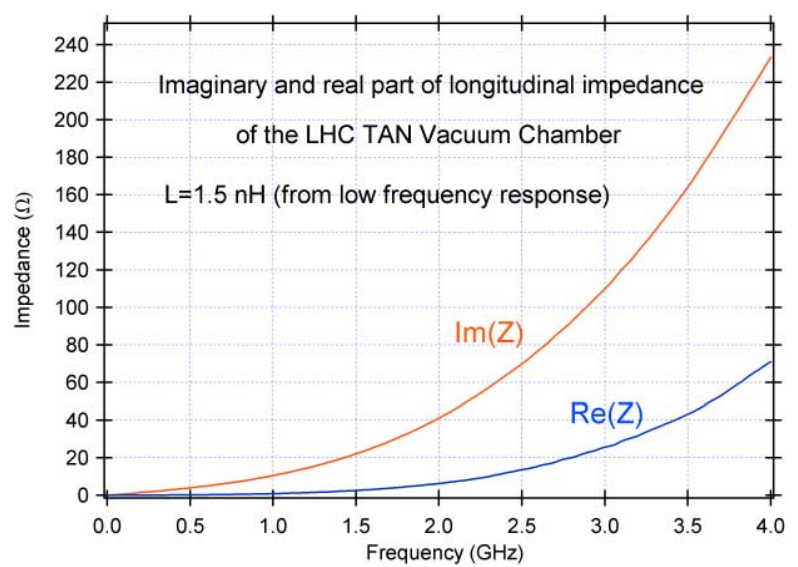

Figure 6: Longitudinal impedance of the TAN chamber calculated using the MAFIA in time domain.

Measurement from the low frequency response of the impedance gives the inductance to be $1.5 \mathrm{nH}$.

For 4 TANs, $\left(Z_{L} / n\right)=0.212 \mathrm{~m} \Omega$, and is small compared to the impedance budget [3].

\section{A RECTANGULAR WEDGE}

To inspect possible trapped modes, a 3D rectangular wedge model is built in comparison with the studies conducted at the CERN [4]. The model is shown in Figure 7 where two square pipes are being smoothly transitioned and combined to a big square pipe.

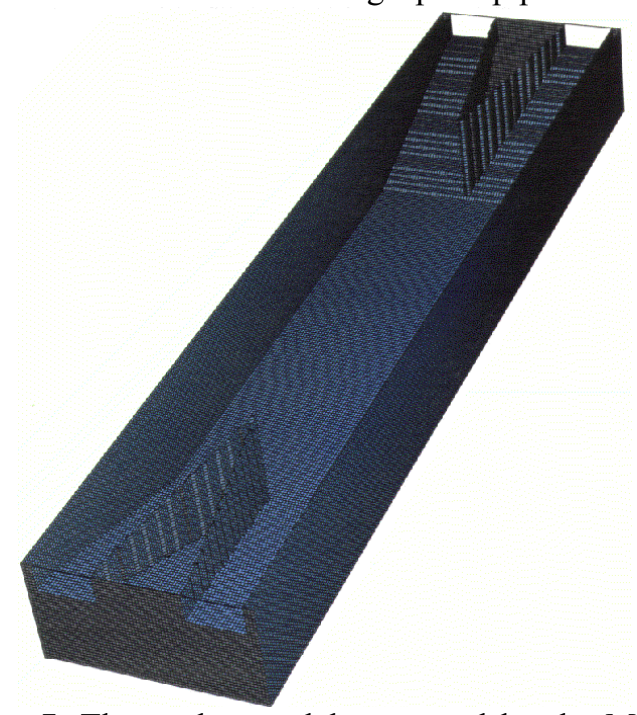

Figure 7: The wedge model generated by the MAFIA used for studying the trapped modes. Dimensions for the wedge: the small pipes: $5.2 \mathrm{~cm} \mathrm{X} 5.2 \mathrm{~cm}$, the big pipe: 18 $\mathrm{cm} \mathrm{X} 18 \mathrm{~cm}$.

MAFIA simulations have been carried out in both the frequency and time domain on the wedge model. Simulation results in the frequency and time domains agree very well with and without trapped mode cases. A mode may be trapped at the sharp edge of the wedge, and a $\mathrm{TM}_{11}$, instead of regular $\mathrm{TE}_{01}$ waveguide mode, is excited as shown in Figure 8. Both the frequency and time domain analyses show this mode is at near $1 \mathrm{GHz}$ while the cutoff of the big rectangular pipe is $830 \mathrm{MHz}$ for $\mathrm{TE}_{01}$ mode and $1.2 \mathrm{GHz}$ for $\mathrm{TM}_{11}$ mode explaining why this mode is trapped. Both the time and frequency simulations show that the frequency of the trapped mode moves higher by shortening (cutting) the sharp wedge edge, and eventually disappeared when the frequency is higher than the $\mathrm{TM}_{11}$ cutoff.

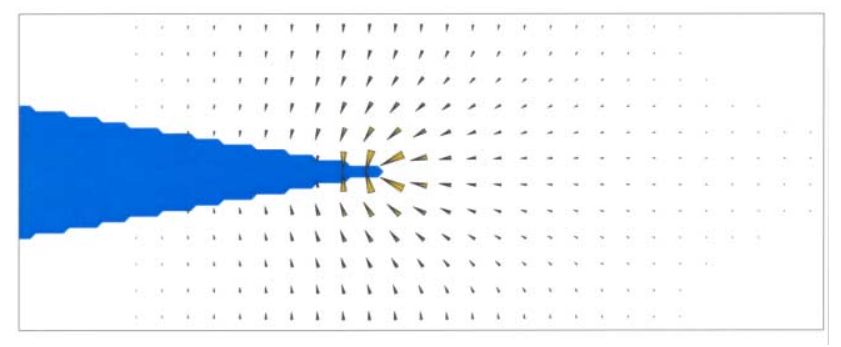

Figure 8: A trapped mode is found to be at frequency $1 \mathrm{GHz}$, the electric field distribution shown above is from the MAFIA simulation in the frequency domain (2D top view of the wedge).

\section{CONCLUSION}

Good agreements are achieved between the time and frequency domain simulations on the TAN vacuum chamber. No trapped mode is found in the TAN chamber. The longitudinal impedance is very small compared to the total impedance budget. Transverse impedance can be calculated using the P-W theory, but needs to be studied and computed carefully due to the asymmetric geometry of the TAN chamber.

\section{REFERENCES}

[1] W. Turner, et al, "Absorbers for the high luminosity insertions of the LHC", EPAC 1998

[2] A. Chao, M. Tigner, "Handbook of Accelerator Physics and Engineering", World Scientific (1998)

[3] F. Ruggiero, "Single-beam collective effects in the LHC", Particle Accelerators, Vol. 50, pp. 83-104 (1995)

[4] D. Brandt, et al, "Impedance of the LHC recombination chambers", LHC Project Note 254. 